\title{
Communicating Reaction Systems with Direct Communication
}

\author{
Erzsébet Csuhaj-Varjú[0000-0002-2773-2944] and Pramod Kumar Sethy \\ Department of Algorithms and Their Applications \\ Faculty of Informatics, Eötvös Loránd University ELTE \\ Budapest, Hungary \\ $\{$ csuhaj, pksethy\}@inf .elte.hu
}

\begin{abstract}
We introduce and examine two variants of networks of reaction systems, called communicating reaction systems with direct communication, where the reaction systems send products or reactions to each other. We show that these types of networks of reaction systems can be obtained by simple mappings from single reaction systems. We also discuss some aspects of communication within these networks, and suggest open problems for future research.
\end{abstract}

\section{Introduction}

The theory of reaction systems has been a vivid research area recently. The concept of a reaction system was introduced by A. Ehrenfeucht and G. Rozenberg as a formal model of interactions between biochemical reactions. The interested reader is referred to [8] for the original motivation. The main idea of the authors was to model the behavior of biological systems in which a large number of individual reactions interact with each other.

A reaction system consists of a finite set of objects that represent chemicals and a finite set of triplets that represent chemical reactions. Each reaction consists of three nonempty finite sets: the set of reactants, the set of inhibitors, and the set of products. The set of reactants and the set of inhibitors are disjoint. Let $T$ be a set of reactants. A reaction is enabled for $T$ and it can be performed if all of its reactants are present in $T$ and none of its inhibitors is in $T$. When the reaction is performed, then the set of its reactants is replaced by the set of its products. All enabled reactions are applied in parallel. The final set of products is the union of all sets of products that were obtained by the reactions that were enabled for $T$. For further details on reaction systems consult [9].

Reaction systems (R systems) are qualitative models, opposed to P systems (membrane systems) that are quantitative ones. The model of reaction systems focuses only on the presence or absence of the chemical species, and does not consider their amounts. Multiple reactions that have common reactants do not interfere. All of the reactions that are enabled at a certain step are performed simultaneously. Another feature of reaction systems which makes them different from other bio-inspired computational models, as for example, $\mathrm{P}$ systems, is the 
lack of permanency: the state of the system consists of only products of those reactions that took place in the last step. Those reactants that were not involved in any reaction disappear from the system. This property is widely used in the theory.

$\mathrm{R}$ systems have been studied in detail over the last 16 years. One interesting topic of their study is the theory of networks of reaction systems [4]. Such a construct is a virtual graph with a reaction system in each node. The reaction systems are defined over the same background set and work in a synchronized manner, governed by the same clock. After performing the reactions enabled for the current set of reactants at a node, certain products from other nodes can be added to the set of products at the node. The nodes, thus the reaction systems interact with each other using distribution and communication protocols. The set of products of each reaction system in the network forms a part of the environment of the network. Important ideas and results on these constructs can be found in $[3,4]$.

In this paper we introduce the concept of communicating $\mathrm{R}$ systems with two variants of direct communication (cdcR systems, for short). These constructs are particular variants of networks of reaction systems [4]. Such a system consists of a finite set of extended versions of reactions defined over the same background set. These extended reaction systems (the components of the cdcR system), in addition to performing standard reactions, communicate either products or reactions to certain predefined target components. In the case of product communication, the products are associated with targets, i.e. labels of components which the product is sent to. In the case of reaction communication, each reaction is associated with a set of targets, labels of components. In this case, after performing the reaction, it is communicated to the target component. We note that the sender component can also be the target component. In both cases, after performing the reactions and the communication, the system performs a new transition. Communication is direct in these systems since the target of the product or the reaction to be communicated is explicitly given together with the cdcR system. We prove that for every cdcR system using any of the two types of direct communication (product or reaction), a reaction system can be constructed which simulates, up to some simple mapping(s), the given cdcR system. That is, these reaction systems provide representations of cdcR systems. We also discuss communication within the network, define static and active communication links, graphs, and describe how to represent active communication links and graphs of the cdcR systems under operation. We also compare the two communication variants. Finally, we provide conclusions and suggestions for future research.

\section{Preliminaries}

For basic notions of formal language and computation theory, the reader is referred to [11]. 
The set of all strings over an alphabet $V$ is denoted by $V^{*}$, the set of nonempty strings by $V^{+}$. The empty string is denoted by $\lambda$ and $|w|$ denotes the length of string $w$. A language $L$ is a subset of $V^{*}$.

We recall the notions concerning reaction systems; most of them are taken from $[8,9]$. Some notations slightly differ from the standard ones; these changes are for technical reasons.

Definition 1. Let $S$ be a finite nonempty set; $S$ is called the background set. A reaction $\rho$ over $S$ is a triplet $\left(R_{\rho}, I_{\rho}, P_{\rho}\right)$ where $R_{\rho}, I_{\rho}, P_{\rho}$ are nonempty subsets of $S$ such that $R_{\rho} \cap I_{\rho}=\emptyset$.

Sets $R_{\rho}, I_{\rho}, P_{\rho}$ are called the sets of reactants, inhibitors, and products of $\rho$, respectively.

For convenience, reaction $\rho$ will be given in the form $\rho:\left(R_{\rho}, I_{\rho}, P_{\rho}\right)$ in the sequel.

We consider now the effect of a reaction in a specific state of a reaction system; states are finite sets of entities.

Definition 2. A reaction system is an ordered pair $\mathcal{A}=(S, A)$, where $S$ is a background set and $A$ is a finite nonempty set of reactions over $S$.

Thus, a reaction system $\mathcal{A}$ is simply a set of reactions. In specifying $A$, we also give its background set $S$.

Definition 3. Let $S$ be a background set, $T \subseteq S, \rho:\left(R_{\rho}, I_{\rho}, P_{\rho}\right)$ be a reaction over $S$, and let $A$ be a finite set of reactions over $S$. Then

1. $\rho$ is enabled for $T$ if $R_{\rho} \subseteq T$ and $I_{\rho} \cap T=\emptyset$;

2. the result of applying $\rho$ to $T$, denoted by $\operatorname{res}_{\rho}(T)$, equals $P_{\rho}$ if $\rho$ is enabled for $T$ and $\emptyset$ otherwise;

3. the result of applying $A$ to $T$, denoted by $\operatorname{res}_{A}(T)$, is $\bigcup_{\rho \in A} \operatorname{res}_{\rho}(T)$.

Thus, reaction $\rho$ is enabled for $T$ if $T$ contains all of the reactants of $\rho$ and none of its inhibitors. If $\rho$ is enabled for $T$, then its product will be a subset of the successor set of reactants. For $T \subseteq S$, en $A(T)$ denotes the set of reactions of $A$ that are enabled for $T$. Notice that res $_{A}$ defines a function on $2^{S}$, called the result function.

Definition 4. The state sequence of a reaction system $\mathcal{A}$ with initial state $T$ is given by successive iterations of the result function:

$$
\left(\operatorname{res}_{\mathcal{A}}^{n}(T)\right)_{n \in N}=\left(T, \operatorname{res}_{\mathcal{A}}(T), \operatorname{res}_{\mathcal{A}}^{2}(T), \ldots\right) .
$$

Since the background set of a reaction system is finite, the state space is also finite; thus, every state sequence is either finite or ultimately periodic. 


\section{Communicating Reaction Systems with Direct Communication}

We introduce the concept of communicating $\mathrm{R}$ systems (cdcR systems) with two variants of direct communication. The concept is strongly related to the notion of a network of $\mathrm{R}$ systems [4] and it has been inspired by several variants of bio-inspired networks of language generating devices $[6,5,7]$. A cdcR system consists of a finite number of components, each component is a finite set of extended variants of reactions. Every component is defined over the same background set. The components, in addition to performing standard reactions, communicate products or reactions, according to the used protocol, to certain predefined target components. The components of the cdcR system work in a synchronized manner, governed by the same clock. In the case of product communication, the products are associated with targets, i.e. with the label of the component which the product is sent to. In the case of reaction communication, each reaction is associated with a set of targets, labels of a component. In this case, after performing the reaction, it is sent to the target components. We note that the target component can also be the sender component. In both cases, after performing the reactions and the communication, the system performs a new transition, i.e. the procedure is repeated. The reader may easily see that the targets define direct communication between the components. We show that for every cdcR system using any of the two types of communication a standard $\mathrm{R}$ system can be constructed which provides a representation of the given cdcR system; the operation of the two systems correspond to each other.

\subsection{Communication by products}

We first define the notion of a cdcR system communicating by products.

Definition 5. A cdcR system communicating by products (a cdcR(p) system, for short), of degree $n, n \geq 1$, is an $(n+1)$-tuple $\Delta=\left(S, A_{1}, \ldots, A_{n}\right)$, where

- $S$ is a finite nonempty set, the background set of $\Delta$;

- $A_{i}, 1 \leq i \leq n$, is the ith component of $\Delta$, where

- $A_{i}$ is a finite nonempty set of extended reactions of type pc (pc-reactions, for short).

- Each pc-reaction $\rho$ of $A_{i}$ is of the form $\rho:\left(R_{\rho}, I_{\rho}, \Pi_{\rho}\right)$, where $R_{\rho}$ and $I_{\rho}$ are nonempty subsets of $S, R_{\rho} \cap I_{\rho}=\emptyset$, and $\Pi_{\rho} \subseteq P_{\rho} \times\{1, \ldots, n\}$ is a nonempty set with $P_{\rho}$ being a nonempty subset of $S . R_{\rho}, I_{\rho}, \Pi_{\rho}$ are called the set of reactants, the set of inhibitors, and the set of products with targets. A pair $(b, j), 1 \leq j \leq n$ in $\Pi_{\rho}$ means that product $b \in S$ is communicated to component $A_{j}$.

The term $p c$-reaction means that the reaction communicates products.

We extend notions and notations concerning reaction systems to $\operatorname{cdcR}(\mathrm{p})$ systems. If it is clear from the context, for singleton sets $\{\rho\}$ we use notation $\rho$. 
A pc-reaction $\rho:\left(R_{\rho}, I_{\rho}, \Pi_{\rho}\right)$ is enabled for the set $U \subseteq S$ if $R_{\rho} \subseteq U$ and $I_{\rho} \cap U=\emptyset$ as in case of standard reaction systems; this fact is denoted by $e n_{\rho}(U)$. Let $U \subseteq S$ be a set of reactants and let $\rho$ be a $p c$-reaction at component $A_{i}$. Then we define $\operatorname{res}_{\rho}(U)=\left\{b \mid(b, i) \in \Pi_{\rho}\right\}$ if $\operatorname{en}_{\rho}(U)$ and $\operatorname{res}_{\rho}(U)=\emptyset$ otherwise.

Let $\Delta=\left(S, A_{1}, \ldots, A_{n}\right)$ be a $\operatorname{cdcR}(\mathrm{p})$ system and let $U \subseteq S$. We define $\operatorname{res}_{A_{i}}(U)=\left\{b \mid(b, i) \in \Pi_{\rho}, \rho \in A_{i}, e n_{\rho}(U)\right\}$ if at least one pc-reaction in $A_{i}$ is enabled for $U$ and $\operatorname{res}_{A_{i}}(U)=\emptyset$ otherwise.

$\operatorname{cdcR}(\mathrm{p})$ systems operate by transitions, i.e. by changing their states. A state of a $\operatorname{cdcR}(\mathrm{p})$ systems $\Delta=\left(S, A_{1}, \ldots, A_{n}\right)$ is an $n$-tuple $\left(D_{1}, \ldots, D_{n}\right)$ where $D_{i} \subseteq S, 1 \leq i \leq n ; D_{i}$ is called the state of component $A_{i}, 1 \leq i \leq n$. Notice that $D_{i}$ can be the empty set.

A transition in $\Delta$ means that every component of the $\operatorname{cdcR}(\mathrm{p})$ system performs all of its enabled $p c$-reactions on the current set of reactants and then communicates the obtained products to their target components, indicated in the corresponding $p c$-reaction. It is important to note that the same object (product) can be communicated to a component from several components and by several $p c$-reactions.

The sequence of transitions starting with an initial state forms a state sequence of $\Delta$. Notice that by the definition of the $p c$-reactions, for a given initial state there is only one state sequence of $\Delta$, i.e. for a given initial state, the sequence of transitions is deterministic.

Definition 6. Let $\Delta=\left(S, A_{1}, \ldots, A_{n}\right), n \geq 1$, be a $c d c R(p)$ system.

The sequence $\bar{D}_{0}, \ldots, \bar{D}_{j}, \ldots$ is called the state sequence of $\Delta$ starting with initial state $\bar{D}_{0}$ if the following conditions are met:

For every $\bar{D}_{j}, j \geq 0$ where $\bar{D}_{j}=\left(D_{1, j} \ldots, D_{i, j}, \ldots, D_{n, j}\right), 1 \leq i \leq n$ it holds that $\bar{D}_{j+1}=\left(D_{1, j+1} \ldots, D_{i, j+1}, \ldots, D_{n, j+1}\right)$ with

$D_{i, j+1}=\cup_{1 \leq k \leq n} \operatorname{Com}_{k \rightarrow i}\left(\operatorname{res}_{A_{k}}\left(D_{k, j}\right)\right)$ where $\operatorname{Com}_{k \rightarrow i}\left(\operatorname{res}_{A_{k}}\left(D_{k, j}\right)\right)=\{b \mid$ $\left.(b, i) \in \Pi_{\rho}, \rho:\left(\bar{R}_{\rho}, I_{\rho}, \Pi_{\rho}\right) \in e n_{A_{k}}\left(D_{k, j}\right)\right\}$.

Sequence $D_{i, 0}, D_{i, 1}, \ldots$ is said to be the state sequence of component $A_{i}$ of $\Delta, 1 \leq i \leq n$.

Notice that the state sequence does not end if $\operatorname{res}_{A_{i}}\left(D_{i, j}\right)$ is the empty set, since products can be communicated to the component in some later step.

Let $\Delta=\left(S, A_{1}, \ldots, A_{n}\right), n \geq 1$, be a $\operatorname{cdcR}(\mathrm{p})$ system and let $\bar{D}_{0}, \bar{D}_{1} \ldots$ be the state sequence of $\Delta$ starting with $\bar{D}_{0}$. Then every pair $\left(\bar{D}_{i}, \bar{D}_{i+1}\right), i \geq 0$ is said to be a transition in $\Delta$ and is denoted by $\bar{D}_{i} \Longrightarrow \bar{D}_{i+1}$.

We give an example for $\operatorname{cdcR}(\mathrm{p})$ systems.

Example 1. Let $\Delta=\left(S, A_{1}, A_{2}, A_{3}\right)$ be a cdcR(p) system where $S=\{a, b, c, d\}$ and components $A_{1}, A_{2}$ and $A_{3}$ are defined as follows. Let

$$
\begin{aligned}
& A_{1}=\left\{\rho_{1}:(\{a, b\},\{d\},\{(a, 2)\}), \rho_{2}:(\{b\},\{d\},\{(b, 2)\})\right\}, \\
& A_{2}=\left\{\rho_{3}:(\{a, b\},\{c\},\{(c, 3)\}), \rho_{4}:(\{a\},\{c\},\{(a, 3)\})\right\}, \\
& A_{3}=\left\{\rho_{5}:(\{a, c\},\{b\},\{(a, 1)\}), \rho_{6}:(\{a\},\{d\},\{(b, 1)\})\right\} .
\end{aligned}
$$


Let $\bar{D}_{0}$, the initial state of $\Delta$ be given as $\bar{D}_{0}=(\{a, b\},\{a, b\},\{a, c\})$. Then component $A_{1}$ performs both of its pc-reactions, $\rho_{1}$ and $\rho_{2}$, and communicates products $a$ and $b$ to component $A_{2}$. Similarly, $A_{2}$ performs both of its $p c$ reactions, $\rho_{3}$ and $\rho_{4}$, and communicates products $c$ and $a$ to component $A_{3}$. As in the previous two cases, $A_{3}$ also performs both of its $p c$-reactions, $\rho_{5}$ and $\rho_{6}$. It communicates products $a$ and $b$ to component $A_{1}$. Thus, the new state of $\Delta$ will be $\bar{D}_{1}=(\{a, b\},\{a, b\},\{a, c\})$, the same as $\bar{D}_{0}$.

If we change $p c$-reaction $\rho_{3}$ to $\rho_{3}^{\prime}$, where $\rho_{3}^{\prime}:(\{c\},\{a, b\},\{(c, 3)\})$, then only pc-reaction $\rho_{4}$ is enabled on $\{a, b\}$. Thus, after performing $\rho_{4}$ only product $a$ is communicated to $A_{3}$. Thus, the new state of $\Delta$ in this case will be $(\{a, b\},\{a, b\},\{a\})$.

Next we show that every cdcR(p) system can be represented by an R system which provides a simulation as well in the following sense: the state sequences of the components of the cdcR(p) system can be obtained by simple mappings from the state sequence of the $R$ system.

Theorem 1. Let $\Delta=\left(S, A_{1}, \ldots, A_{n}\right), n \geq 1$, be a $c d c R(p)$ system and let $\bar{D}_{0}=\left(D_{1,0}, \ldots, D_{n, 0}\right)$ be initial state of $\Delta$. We can give a reaction system $\mathcal{A}=$ $\left(S^{\prime}, A^{\prime}\right)$, initial state $W_{0}$ of $\mathcal{A}$, and mappings $h_{i}: 2^{S^{\prime}} \rightarrow 2^{S}$ such that for each $i, 1 \leq i \leq n$, the state sequence $D_{i, 0}, D_{i, 1}, \ldots$ of component $A_{i}$ of $\Delta$ is equal to the sequence $h_{i}\left(W_{0}\right), h_{i}\left(W_{1}\right), \ldots$, where $W_{0}, W_{1}, \ldots$ is the state sequence of $\mathcal{A}$ starting from initial state $W_{0}$.

Proof. To prove the statement, we first define the components of $\mathcal{A}$. Let $S^{\prime}=$ $\{[x, i] \mid x \in S, 1 \leq i \leq n\}$ be the background set of $\mathcal{A}$. For every $i, 1 \leq i \leq n$ let $S_{i}^{\prime}=\{[x, i] \mid x \in S\}$.

For any $p c$-reaction $\rho:\left(R_{\rho}, I_{\rho}, \Pi_{\rho}\right)$ of component $A_{i}, 1 \leq i \leq n$, we define reaction $\rho^{\prime}:\left(R_{\rho^{\prime}}, I_{\rho^{\prime}}, P_{\rho^{\prime}}\right)$ of $\mathcal{A}$ as follows: $R_{\rho^{\prime}}=\left\{[x, i] \mid x \in R_{\rho}\right\}, I_{\rho^{\prime}}=\{[y, i] \mid$ $\left.x \in I_{\rho}\right\}, P_{\rho^{\prime}}=\left\{[x, k] \mid(x, k) \in \Pi_{\rho}, 1 \leq k \leq n\right\} . \mathcal{A}$ has no more reactions. It can immediately be seen that every reactant $[x, i]$ of $\mathcal{A}$ represents a reactant $x$ in $S$ that can be found at component $A_{i}$, and reversely. Thus, $\Delta$ and $\mathcal{A}$ correspond to each other, since by definition any reaction $\rho^{\prime}:\left(R_{\rho^{\prime}}, I_{\rho^{\prime}}, P_{\rho^{\prime}}\right)$ of $\mathcal{A}$ where each element of $R_{\rho^{\prime}}, I_{\rho^{\prime}}$ is of the form $[x, i]$ corresponds to a pc-reaction $\rho:\left(R_{\rho}, I_{\rho}, \Pi_{\rho}\right)$ of component $A_{i}$, and reversely.

Let $W_{0}=\left\{[x, i] \mid x \in D_{i, 0}, 1 \leq i \leq n\right\}$ be the initial state of $\mathcal{A}$. It is easy to see that elements of $W_{0}$ correspond to elements of the initial states of the components of $\Delta$.

Let us define for $i, 1 \leq i \leq n$, mapping $h_{i}: 2^{S^{\prime}} \rightarrow 2^{S}$ as follows. Let $U \subseteq S^{\prime}$. If $U \cap S_{i}^{\prime} \neq \emptyset$, then let $h_{i}(U)=\{x \mid[x, i] \in U\}$, otherwise let $h_{i}(U)=\emptyset$.

We prove that the state sequence of component $A_{i}$ of $\Delta$ starting from initial state $D_{i, 0}$ corresponds to the state sequence of $\mathcal{A}$ starting from $W_{0}$. For $j=0$ and for any fixed $i, i \in\{1, \ldots, n\}, D_{i, 0}=h_{i}\left(W_{0}\right)$, thus the statement for $j=0$ holds. Suppose now that the statement holds for $l$, where $l \geq 1$, i.e. $D_{i, l}=h_{i}\left(W_{l}\right)$. We show that $D_{i, l+1}=h_{i}\left(W_{l+1}\right)$ holds as well. The set of reactants $D_{i, l+1}$ is the union of two sets of reactants $U_{i, l+1}$ and $V_{i, l+1} . U_{i, l+1}$ consists of all products that are obtained by all enabled reactions of $A_{i}$ performed on $D_{i, l}$ and which 
products do not leave the component $A_{i}$, i.e. which should be communicated to $A_{i} . V_{i, l+1}$ consists of all products of all enabled reactions performed on some $D_{k, l}$ which products leave component $A_{k}, k \neq i$. (Notice that the two sets $U_{l+1}$ and $V_{i, l+1}$ can have joint elements.) Since $D_{i, l+1}=\cup_{1 \leq k \leq n} \operatorname{Com}_{k \rightarrow i}\left(\operatorname{res}_{A_{k}}\left(D_{k, l}\right)\right)$ where $C \operatorname{mom}_{k \rightarrow i}\left(\operatorname{res}_{A_{k}}\left(D_{k, l}\right)\right)=\left\{b \mid(b, i) \in P_{\rho} \times\{1, \ldots, n\}, \rho=\left(R_{\rho}, I_{\rho}, \Pi_{\rho}\right) \in\right.$ $\left.e n_{A_{k}}\left(D_{k, l}\right)\right\}$ and each $p c$-reaction $\rho=\left(R_{\rho}, I_{\rho}, \Pi_{\rho}\right)$ of component $A_{i}$ corresponds to exactly one reaction $\rho^{\prime}:\left(R_{\rho^{\prime}}, I_{\rho^{\prime}}, P_{\rho^{\prime}}\right)$ of $\mathcal{A}$ and reversely, where $R_{\rho^{\prime}}=\{[x, i] \mid$ $\left.x \in R_{\rho}\right\}, I_{\rho^{\prime}}=\left\{[y, i] \mid x \in I_{\rho}\right\}, P_{\rho^{\prime}}=\left\{[x, k] \mid(x, k) \in \Pi_{\rho}, 1 \leq k \leq n\right\}$, it can be seen that $D_{i, l+1}=h_{i}\left(W_{l+1}\right)$ holds. This implies that the statement of the theorem holds.

In the sequel, we also call reaction system $\mathcal{A}$ the flattened reaction system of $\Delta$ or a flattened version of $\Delta$. Notice that a $\operatorname{cdcR}(\mathrm{p})$ system is allowed to have only one component, thus the use of the term flattened version is justified.

Definition 7. Let $\Delta=\left(S, A_{1}, \ldots, A_{n}\right), n \geq 1$, be a $c d c R(p)$ system. Let reaction system $\mathcal{A}=\left(S^{\prime}, A^{\prime}\right)$ be defined as follows. Let $S^{\prime}=\{[x, i] \mid x \in S, 1 \leq i \leq$ $n\}$ be the background set of $\mathcal{A}$. For any pc-reaction $\rho:\left(R_{\rho}, I_{\rho}, \Pi_{\rho}\right)$ of component $A_{i}$, we define reaction $\rho^{\prime}:\left(R_{\rho^{\prime}}, I_{\rho^{\prime}}, P_{\rho^{\prime}}\right)$ of $\mathcal{A}$ with $R_{\rho^{\prime}}=\left\{[x, i] \mid x \in R_{\rho}\right\}$, $I_{\rho^{\prime}}=\left\{[y, i] \mid x \in I_{\rho}\right\}, P_{\rho^{\prime}}=\left\{[x, k] \mid(x, k) \in \Pi_{\rho}, 1 \leq k \leq n\right\}$. No other reaction is in $A^{\prime}$. Then $\mathcal{A}$ is called the flattened reaction system of $\Delta$.

Based on the proof of Theorem 1 some observations can be made. We present the next statement without proof, since it is a direct consequence of Theorem 1 and its proof.

Corollary 1. Let $\Delta=\left(S, A_{1}, \ldots, A_{n}\right), n \geq 1$, be a $c d c R(p)$ system and let $\mathcal{A}=\left(S^{\prime}, A^{\prime}\right)$ be an $R$ system given as in Theorem 1. Furthermore, let $\bar{D}_{0}$ be the initial state of $\Delta$ and let $W_{0}$ be the initial state of $\mathcal{A}$ given as in the proof of Theorem 1. Then, for $m \geq 0$, a reactant $b \in S$ occurs at component $A_{i}$ in the $m$ th element of the state sequence of $\Delta$ starting with initial state $\bar{D}_{0}$ if and only if reactant $[b, i] \in S^{\prime}$ occurs in the mth element of state sequence of $\mathcal{A}$ starting with initial state $W_{0}$.

In $[12,10]$ the following problem was discussed: For a given reaction system $\mathcal{A}=(S, A)$, a reactant $a \in S$ and $m \geq 2$ the decision problem whether $a$ appears at the $m$ th step of at least one state sequence of $\mathcal{A}$ is called the occurrence problem. Note that any nonempty subset of $S$ can be considered as initial state of $\mathcal{A}$, thus the reaction system may have more than one state sequences. For some fixed values of the parameter $m$, the occurrence problem was shown to be NP-complete [12] and when $m$ is given as input it is a PSPACE-problem [10].

We can formulate the occurrence problem for $\operatorname{cdcR}(\mathrm{p})$ systems as well. For a given $\operatorname{cdcR}(\mathrm{p})$ system $\Delta=\left(S, A_{1}, \ldots, A_{n}\right), n \geq 1$, the problem whether a reactant $a \in S$ occurs at some component $A_{i}$ at the $m$ th element of the state sequence of $\Delta$ starting with some some initial state $\bar{D}_{0}$ is called the occurrence problem of $\operatorname{cdcR}(\mathrm{p})$ systems. By Theorem 1, Corollary 1 and because to any reaction system we can construct a $\operatorname{cdcR}(\mathrm{p})$ system with only one component, 
we may state that the occurrence problem of $\operatorname{cdcR}(\mathrm{p})$ systems for some fixed values of $m$ is NP-complete and it is a PSPACE-problem when $m$ is given as input.

Next we deal with the communication of products within the cdcR(p) system under operation.

Definition 8. Let $\Delta=\left(S, A_{1}, \ldots, A_{n}\right), n \geq 1$, be a cdcR $(p)$ system. The static communication graph of $\Delta$ is a directed graph $\Gamma=(V, E)$, where $V$ is the set of vertices (nodes) labeled with $A_{j}, 1 \leq j \leq n$, and the set of edges $E$ is defined by $E \subseteq \bar{A} \times \bar{A}$, where $\bar{A}=\left\{A_{1}, \ldots, A_{n}\right\}$ and $\left(A_{i}, A_{j}\right) \in E$ if and only if there is a pc-reaction $\rho:\left(R_{\rho}, I_{\rho}, \Pi_{\rho}\right)$ in $A_{i}$ such that $\Pi_{\rho}$ contains an element $(b, j)$.

That is, from node $A_{i}$ there is a directed edge to node $A_{j}$ if and only if component $A_{i}$ of $\Delta$ has at least one $p c$-reaction that communicates at least one product to component $A_{j}$.

Definition 9. Let $\Delta=\left(S, A_{1}, \ldots, A_{n}\right), n \geq 1$, be a $c d c R(p)$ system. Let $\bar{D}_{0}$ be an initial state of $\Delta$ and let $t_{l}: \bar{D}_{l} \Longrightarrow \bar{D}_{l+1}, l \geq 0$ be a transition in the state sequence $\sigma: \bar{D}_{0}, \bar{D}_{1}, \ldots, \bar{D}_{l}, \ldots$ of $\Delta$. If under transition $t_{l}$, at component $A_{i}$ at least one reaction is performed that communicates at least one product to component $A_{j}, 1 \leq i, j \leq n$, then we say that there is an active communication link from component $A_{i}$ to component $A_{j}$ under transition $t_{l}: \bar{D}_{l} \Longrightarrow \bar{D}_{l+1}$ in state sequence $\sigma$.

The active communication graph $\Gamma_{t r_{l}}=\left(V, E_{t r_{l}}\right)$ of $\Delta$ under transition $t r_{l}$ in $\sigma$ is defined as follows: $V$ is given as for $\Gamma$ and $E_{t r_{l}}$ consists of all edges $\left(A_{i}, A_{j}\right), 1 \leq i, j \leq n$ in $E$ such that there is an active communication link from component $A_{i}$ to component $A_{j}$ under transition $\operatorname{tr}_{l}: \bar{D}_{l} \Longrightarrow \bar{D}_{l+1}$.

Notice that the active communication graph is associated to a transition. Thus, if $\sigma: \bar{D}_{0}, \bar{D}_{1}, \ldots$ of $\Delta$ is the state sequence of $\Delta$ starting from initial state $\bar{D}_{0}$, then $\sigma$ defines a sequence of graphs $\Gamma_{t r_{i}}, i \geq 1$, where $\Gamma_{t r_{i}}$ is the active communication graph associated to transition $t r_{i}, t r_{i}: \bar{D}_{i-1} \Longrightarrow \bar{D}_{i}$.

In the following we provide a representation of communication graphs (static and active) of $\operatorname{cdcR}(\mathrm{p})$ systems. In the proof of Theorem 1, we assigned to each product $b$ of $\operatorname{cdcR}(\mathrm{p})$ system $\Delta$ a location, i.e. the number (label) of the component where the reactant is currently located. Thus, we used products of the form $[b, i]$ instead of $b$. This idea is extended in the following manner. In addition to the current place, the symbol describing the product will also code its previous location, the component from which it was communicated to its recent location. Thus, we will use symbols of the form $[b, i, j]$ meaning that a product $b$ from component $A_{i}$ is/was sent to component $A_{j}$. Using this variant of flattening the $\operatorname{cdcR}(\mathrm{p})$ system, we find a method for tracking active communication links associated to transitions in every given state sequence in $\Delta$.

Theorem 2. Let $\Delta=\left(S, A_{1}, \ldots, A_{n}\right), n \geq 1$ be a cdcR(p) system and let $\bar{D}_{0}$ be initial state of $\Delta$. Let $\mathcal{A}=\left(S^{\prime}, A^{\prime}\right)$ be a reaction system and let $W_{0}$ be initial state of $\mathcal{A}$ where 
- $S^{\prime}=\left\{[x, i],[x, i, k]^{\prime} \mid x \in S, 1 \leq i, k \leq n\right\}$, and

- $A^{\prime}$ consists of the following reactions.

- For any pc-reaction $\rho:\left(R_{\rho}, I_{\rho}, \Pi_{\rho}\right)$ of component $A_{i}$, there is reaction $\rho^{\prime}:\left(R_{\rho^{\prime}}, I_{\rho^{\prime}}, P_{\rho^{\prime}}\right)$ in $A^{\prime}$ with $R_{\rho^{\prime}}=\left\{[x, i] \mid x \in R_{\rho}\right\}, I_{\rho^{\prime}}=\{[y, i] \mid y \in$ $\left.I_{\rho}\right\}, P_{\rho^{\prime}}=\left\{[x, i, k]^{\prime} \mid(x, k) \in \Pi_{\rho}, 1 \leq k \leq n\right\}$.

- For every $x \in S$ and $1 \leq i, k \leq n$ there is a reaction $\rho_{[x, i, k]^{\prime}}:\left(\left\{[x, i, k]^{\prime}\right\},\{[x, k]\},\{[x, k]\}\right)$ in $A^{\prime}$.

- $W_{0}$ consists of all reactants $[x, h]$ where $x \in S$ and $[x, h]$ is an element of $D_{h, 0}, 1 \leq h \leq n$.

Then for any $j, j \geq 0$, under transition $\operatorname{tr}: \bar{D}_{j} \Longrightarrow \bar{D}_{j+1}$ in the state sequence $\bar{D}_{0}, \bar{D}_{1}, \ldots, \bar{D}_{j}, \bar{D}_{j+1}, \ldots$ of $\Delta$ there is an active communication link from component $A_{i}$ to component $A_{k}$ of $\Delta$ if and only if for some $x \in S$ there is a reactant $[x, i, k]^{\prime} \in S^{\prime}$ which is a product of an enabled reaction of $\mathcal{A}$ on $W_{2 j}$ in transition $W_{2 j} \Longrightarrow W_{2 j+1}$ of the state sequence $W_{0}, W_{1}, \ldots$ of $\mathcal{A}$.

This statement can be proven by modifying the proof of Theorem 1, we leave the details to the reader.

\subsection{Communication by reactions}

Under operation, the architecture of the $\mathrm{cdcR}(\mathrm{p})$ system remains unchanged in the sense that the set of reactions of the component does not change. An interesting question is the following: What can we say about communicating reaction systems where the current sets of reactions of the components are allowed to change from state to state. One possible variant of this model is where the (successfully) performed reactions can be communicated to the other components and if a reaction is available at some component in some state then it had to be performed at some component in the previous state (except the case of the initial state). This type of cdcR systems can be considered as a dynamically evolving system and represents a communication model where rules and not data are communicated.

Definition 10. A cdcR system communicating by reactions (a cdcR( $r$ ) system, for short) of degree $n, n \geq 1$, is a triplet $\Delta=(n, S, \mathcal{R})$ where

- $n$ is the number of components,

- $S$ is a finite nonempty set, called the background set of $\Delta$,

$-\mathcal{R}$ is a finite nonempty set of extended reactions of type rc (rc-reactions, for short), where

- each rc-reaction is of the form $\rho:\left(R_{\rho}, I_{\rho}, P_{\rho}\right) ; \operatorname{target}(\rho)$,

- $R_{\rho}, I_{\rho}, P_{\rho}$ are nonempty subsets of $S$, the set of reactants, the set of inhibitors, and the set of products of the rc-reaction, respectively,

- $\operatorname{target}(\rho) \subseteq\{1, \ldots, n\}$ is a nonempty set, the set of indices (labels) of the target components to which the rc-reaction is communicated. 
The components are labeled by numbers $i, 1 \leq i \leq n$.

For an $r c$-reaction $\rho=\left(R_{\rho}, I_{\rho}, P_{\rho}\right)$; $\operatorname{target}(\rho)$, triplet $\left(R_{\rho}, I_{\rho}, P_{\rho}\right)$ is called its core and is denoted by $\operatorname{core}(\rho)$. For a nonempty set $\mathcal{R}^{\prime} \subseteq \mathcal{R}$ we define $\operatorname{core}\left(\mathcal{R}^{\prime}\right)=$ $\left\{\right.$ core $\left.(\rho) \mid \rho \in \mathcal{R}^{\prime}\right\}$. An $r c$-reaction $\rho$ is enabled for a nonempty subset $U$ of $S$ if core $(\rho)$ is enabled for $U$; the result of performing $\rho$ on $U$ means the result of performing core $(\rho)$ on $U$. Notations $e n_{\rho}(U), \operatorname{res}_{\rho}(U)$, and $e n_{R^{\prime}}(U), \operatorname{res}_{R^{\prime}}(U)$ where $\rho$ is an $r c$-reaction and $R^{\prime}$ is a set of $r c$-reaction systems are used in the usual manner.

If no confusion arises, from now on $\rho$ will be called the label of reaction $\rho$ as well.

Next we define the operation of $\operatorname{cdcR}(\mathrm{r})$ systems. These systems work with changing their configurations, i.e. changing the current reaction sets and the current sets of reactants that are at the disposal of the components. While the behavior of $\operatorname{cdcR}(\mathrm{p})$ systems can be represented by the state sequences, in case of $\operatorname{cdcR}(\mathrm{r})$ systems we speak of configuration sequences, since reaction sets are allowed to be changed as well.

Definition 11. Let $\Delta=(n, S, \mathcal{R}), n \geq 1$, be a cdcR(r) system with $n$ components. Let $\bar{C}_{0}$ be the initial configuration of $\Delta$ where $\bar{C}_{0}=\left(\left(A_{1,0}, D_{1,0}\right) \ldots\right.$,

$\left(A_{n, 0}, D_{n, 0}\right)$ ) with $A_{i, 0} \subseteq \mathcal{R}$ (the initial rc-reaction set of component $i$ ) and $D_{i, 0} \subseteq S$ (the initial reactant set of component $\left.i\right), 1 \leq i \leq n$. The pair $\left(A_{i, 0}, D_{i, 0}\right)$ is called the initial configuration of component $i$.

The configuration sequence $\bar{C}_{0}, \bar{C}_{1}, \ldots$ of $\Delta$, where $\bar{C}_{j}=\left(\left(A_{1, j}, D_{1, j}\right) \ldots\right.$, $\left.\left(A_{n, j}, D_{n, j}\right)\right), j \geq 0$, is defined as follows:

For each component $i, 1 \leq i \leq n$, for each $j, j \geq 0$ and for every subsequent configurations $\left(A_{i, j}, D_{i, j}\right),\left(A_{i, j+1}, D_{i, j+1}\right)$ of component $i$ the following hold:

- $A_{i, j+1}=\left\{\rho \in \mathcal{R} \mid i \in \operatorname{target}(\rho), \rho \in A_{k, j}, e n_{\operatorname{core}(\rho)}\left(D_{k, j}\right), 1 \leq k \leq n\right\}$ and

- $D_{i, j+1}=\operatorname{res} \operatorname{core}\left(A_{i, j}\right)\left(D_{i, j}\right)$

That is, after performing the reactions that are enabled for the current reactant sets at the components, the products stay with the components and those reactions that were enabled for the reactant set are communicated. This means that these reactions are added to the reaction sets of their target components. (Notice that the sender component can be a target component as well). The new set of reactions of the component consists of all reactions that were obtained by communication. (Thus, those reactions that were not enabled for the reactant set are erased from the set of reactions of the component.)

We give an example for a cdcR(r) system.

Example 2. Let $\Delta=(3, S, \mathcal{R})$ be a cdcR(r) system where $S=\{a, b, c, d\}$ and $\mathcal{R}$ is defined as follows. Let

$$
\begin{aligned}
\mathcal{R}=\left\{\rho_{1}:(\{a, b\},\{d\},\{a\}) ;\{1,2\},\right. & \\
\rho_{2} & :(\{b\},\{d\},\{b\}) ;\{1,2\}, \\
& \rho_{3}:(\{a, b\},\{c\},\{c\}) ;\{2,3\}, \\
& \rho_{4}:(\{a\},\{c\},\{a\}) ;\{2,3\}, \\
& \rho_{5}:(\{a, c\},\{b\},\{a\}) ;\{3,1\}, \\
& \left.\rho_{6}:(\{a\},\{d\},\{b\}) ;\{3,1\}\right\} .
\end{aligned}
$$


Let the initial configuration of $\Delta, \bar{C}_{0}=\left(\left(A_{1,0}, D_{1,0}\right),\left(A_{2,0}, D_{2,0}\right),\left(A_{3,0}, D_{3,0}\right)\right)$ be given as follows. Let $A_{1,0}=\left\{\rho_{1}, \rho_{2}\right\}, A_{2,0}=\left\{\rho_{3}, \rho_{4}\right\}$, and $A_{3,0}=\left\{\rho_{5}, \rho_{6}\right\}$. Let $D_{1,0}=\{a, b\}, D_{2,0}=\{a, b\}, D_{3,0}=\{a, c\}$, i.e. the same initial states and sets of reactants as in Example 1.

The new configuration $\bar{C}_{1}$ of $\Delta$ will be the following. It can easily be seen that each reaction can be performed at each component, thus the new $r c$-reaction sets will be the following. The first component will have $r c$-reactions $\rho_{1}, \rho_{2}, \rho_{5}, \rho_{6}$, the second component will have $r c$-reactions $\rho_{3}, \rho_{4}, \rho_{1}, \rho_{2}$, and the third component will have $r c$-reactions $\rho_{5}, \rho_{6}, \rho_{3}, \rho_{4}$. The new states will be $\{a, b\},\{a, c\},\{a, b\}$, respectively. Repeating the procedure, the state of the first component will be $\{a, b\}$, the state of the second component will be $\{a, b\}$, and the third component will have state $\{a, b\}$ as well.

As with $\operatorname{cdcR}(\mathrm{p})$ systems, to every $\operatorname{cdcR}(\mathrm{r})$ system $\Delta$ we can construct an $\mathrm{R}$ system $\mathcal{A}$ which represents $\Delta$.

Theorem 3. Let $\Delta=(n, S, \mathcal{R}), n \geq 1$ be a cdcR(r) system of degree $n$, and let $L a b_{R}=\left\{l_{\rho} \mid \rho \in \mathcal{R}\right\}$ be the set of labels associated to the elements of $\mathcal{R} ; L^{2} b_{R}$ and $S$ are disjoint sets.

Let $\sigma=\bar{C}_{0}, \bar{C}_{1}, \ldots$ be the configuration sequence of $\Delta$ starting from initial configuration $\bar{C}_{0}$, where $\bar{C}_{j}=\left(\left(A_{1, j}, D_{1, j}\right) \ldots,\left(A_{n, j}, D_{n, j}\right)\right), j \geq 0$.

We can construct a reaction system $\mathcal{A}=\left(S^{\prime}, A^{\prime}\right)$, give initial state $W_{0}$ of $\mathcal{A}$ and mappings $h_{i}, g_{i}, 1 \leq i \leq n$ such that for every pair $\left(A_{i, j}, D_{i, j}\right), j \geq 0$, in the configuration sequence $\sigma$ it holds that $h_{i}\left(W_{j}\right)=D_{i, j}$ and $g_{i}\left(W_{j}\right)=L a b_{A_{i, j}}$ where $L_{a b} b_{i, j}$ denotes the set of labels of rc-reactions that are elements of $A_{i, j}$ and $W_{0}, W_{1}, \ldots$ is the state sequence of $\mathcal{A}$ starting from $W_{0}$.

Proof. Let us define $\mathcal{A}=\left(S^{\prime}, A^{\prime}\right)$ as follows. Let $S^{\prime}=\left\{[a, i] \mid a \in\left(S \cup L a b_{R}\right), 1 \leq\right.$ $i \leq n\}$. To each $r c$-reaction $\rho:\left(R_{\rho}, I_{\rho}, P_{\rho}\right) ; \operatorname{target}(\rho)$ in $\mathcal{R}$ and for each $i, 1 \leq i \leq$ $n$, we define a reaction $\left(\rho^{\prime}, i\right):\left(\left\{\left[l_{\rho}, i\right]\right\} \cup\left\{[a, i] \mid a \in R_{\rho}\right\},\left\{[b, i] \mid b \in I_{\rho}\right\},\{[c, i] \mid \in\right.$ $\left.\left.P_{\rho}\right\} \cup\left\{\left[l_{\rho}, k\right] \mid k \in \operatorname{target}(\rho)\right\}\right)$.

Let $W_{0}=\bigcup_{i=1}^{n}\left(\left\{\left[l_{\rho}, i\right] \mid l_{\rho} \in L a b_{R}, \rho:\left(R_{\rho}, I_{\rho}, P_{\rho}\right) ; \operatorname{target}(\rho) \in A_{i, 0}\right\} \cup\{[b, i] \mid\right.$ $\left.b \in D_{i, 0}\right\}$.

Let us define mapping $h_{i}: 2^{S^{\prime}} \rightarrow 2^{S}, 1 \leq i \leq n$ as follows. For $U \subseteq S^{\prime}$ with $U \cap S^{\prime} \neq \emptyset$, let $h_{i}(U)=\{x \mid[x, i] \in U\}$, otherwise let $h_{i}(U)=\emptyset$. (Notice that if $U=\{[x, i],[y, j]\}$ where $j \neq i$, then $h_{i}(U)=\{x\}$.) Let mapping $g_{i}$ : $2^{S^{\prime}} \rightarrow 2^{L a b_{R}}, 1 \leq i \leq n$ be defined as follows. For $V \subseteq S^{\prime}$ and $V \cap S^{\prime} \neq \emptyset$ let $g_{i}(V)=\left\{l_{\rho} \mid\left[l_{\rho}, i\right] \in V, V \subseteq L a b_{R}\right\}$, otherwise let $g_{i}(V)=\emptyset$.

By definition, it is obvious that $h_{i}\left(W_{0}\right)=D_{i, 0}$ and $g_{i}\left(W_{0}\right)=L a b_{A_{i, 0}}$, where $L a b_{A_{i, 0}}$ denotes the labels of reactions in $A_{i, 0}$.

Suppose now that for any fixed $i$, and up to certain $j, j \geq 1$ for $\left(A_{i, j}, D_{i, j}\right)$ in the configuration sequence of $\Delta$ it holds that $h_{i}\left(W_{j}\right)=D_{i, j}$ and $g_{i}\left(W_{j}\right)=$ $L a b_{A_{i, j}}$ where $L a b_{A_{i, j}}$ denotes the set of labels of reactions that are elements of $A_{i, j}$, and $W_{j}$ is the $j$ th element in the state sequence of $\delta$ starting from its initial state $W_{0}$. We show now that the statement holds for $j+1$ as well.

Notice that due to the form of the reactions of $\mathcal{A}$, for any $j$, where $j \geq 1$, $\left[l_{\rho}, i\right]$ appears in $W_{j}$ if and only if it was obtained as a product in the previous 
step by some reaction of $\mathcal{A}$. By the reactions of $\mathcal{A}$ this is possible if and only if $\left(R_{\rho}, I_{\rho}, P_{\rho}\right)$ was performed at some component of $\Delta$ and $\rho$ was communicated to component $i$. Thus, reactants of the form $\left[l_{\rho}, i\right]$ in $W_{j}$ and reactions in $A_{i, j}$ correspond to each other. Analogously, any reactant of the form $[b, i]$ occurs in $W_{j}$ if and only if it is an element of $D_{i, j}$. Applying reactions of $\mathcal{A}$ to $W_{j}$, elements of $W_{j+1}$ will be of the form $[\gamma, i]$ and $[c, i]$ where $\gamma \in L a b_{A_{i, j+1}}$ and $c \in S$ meet the previously listed conditions. Notice that labels of reactions of $\Delta$ are reactants of $\mathcal{A}$ that indicate the simulation of a reaction in $\Delta$ with a reaction of $\mathcal{A}$. Thus, that the statement of the theorem holds.

Analogously to Theorem 1, the previous statement has a direct consequence. So the proof is left to the reader.

Corollary 2. Let $\Delta$ be a cdcR(r) system of degree $n, n \geq 1$, and let $\mathcal{A}$ be an $R$ system given as in Theorem 3. Let $\bar{C}_{0}$ be the initial configuration of $\Delta$ and let $W_{0}$ be the initial state of $\mathcal{A}$ given as in Theorem 3. Then rc-reaction $\rho$ occurs at component $i$ in the mth element of state sequence of $\Delta$ starting from $\bar{C}_{0}$ if and only if reactant $\left[l_{\rho}, i\right]$ occurs in the mth element of state sequence of $\mathcal{A}$ starting from $W_{0}$, where $m \geq 1$.

As for cdcR(p) systems, the reaction system $\mathcal{A}$ constructed to cdcR(r) system $\Delta$ in Theorem 3 can be called the flattened reaction system of $\Delta$ and we can formulate an occurrence problem to $\operatorname{cdcR}(\mathrm{r})$ systems as follows. For a given $\operatorname{cdcR}(\mathrm{r})$ system $\Delta=(n, S, \mathcal{R}), n \geq 1$, the problem whether an $r c$-reaction $\rho \in \mathcal{R}$ occurs at the $i$ th component at the $m$ th element of the state sequence of $\Delta$ starting with some initial configuration $\bar{C}_{0}$ is called the occurrence problem of $\operatorname{cdcR}(\mathrm{r})$ systems. By Theorem 3 and Corollary 2, and by $[12,10]$ we may state that the occurrence problem of $\operatorname{cdcR}(\mathrm{r})$ systems for some fixed values of $m$ is NP-complete and it is a PSPACE-problem when $m$ is given as input.

Analogously to $\mathrm{cdcR}(\mathrm{p})$ systems, we define the flattened reaction system of $\operatorname{cdcR}(\mathrm{r})$ systems $\Delta$.

Definition 12. Let $\Delta=(n, S, \mathcal{R}), n \geq 1$ be a cdcR(r) system of degree $n$, and let $L a b_{R}=\left\{l_{\rho} \mid \rho \in \mathcal{R}\right\}$ be a set of labels associated to the elements of $\mathcal{R}$. Let $L a b_{R}$ and $S$ be disjoint sets. Let us define reaction system $\mathcal{A}=\left(S^{\prime}, A^{\prime}\right)$ as follows. Let $S^{\prime}=\left\{[a, i] \mid a \in\left(S \cup L a b_{R}\right), 1 \leq i \leq n\right\}$. To each rc-reaction $\rho:\left(R_{\rho}, I_{\rho}, P_{\rho}\right) ; \operatorname{target}(\rho)$ in $\mathcal{R}$ and for each $i, 1 \leq i \leq n$, we define a reaction $\left(\rho^{\prime}, i\right):\left(\left\{\left[l_{\rho}, i\right]\right\} \cup\left\{[a, i] \mid a \in R_{\rho}\right\},\left\{[b, i] \mid b \in I_{\rho}\right\},\left\{[c, i] \mid \in P_{\rho}\right\} \cup\left\{\left[l_{\rho}, k\right] \mid k \in\right.\right.$ target $(\rho)\})$. $\mathcal{A}$ has no more reactions. Then $\mathcal{A}$ is called the flattened reaction system of $c d c R(r)$ system $\Delta$.

We have shown that both $c d c R(p)$ systems and $c d c R(r)$ systems can be flattened, i.e. we can construct simulating reaction systems to both types of cdcR systems. To obtain the simulating reaction system, either we indicated the location of the reactant or we indicated both the location of the reactant and the location of the reaction in the set of new reactants. In the case of $\operatorname{cdcR}(\mathrm{r})$ systems, we added the labels of $r c$-reactions to the reactant set of the reactions. 
Studying the proofs, the reader may notice that the simulating reaction systems are similar. Based on this observation, we show that to any $\operatorname{cdcR}(\mathrm{r})$ system we can construct a $\operatorname{cdcR}(\mathrm{p})$ system such that there exists a reaction system which is the flattened version of both.

Theorem 4. Let $\Delta=(n, S, \mathcal{R}), n \geq 1$ be a $c d c R(r)$ system of degree $n$ and let $\mathcal{A}$ be the flattened reaction system of $\Delta$ given as in Definition 12 . Then there exists a cdcR $(p)$ system $\Delta^{\prime}$ such that for its flattened reaction system $\mathcal{A}^{\prime}$, given as in Definition $7, \mathcal{A}=\mathcal{A}^{\prime}$ holds.

Proof. Let us consider $\Delta=(n, S, \mathcal{R}), n \geq 1$ and let $L a b_{R}=\left\{l_{\rho} \mid \rho \in \mathcal{R}\right\}$ be a set of labels associated to the elements of $\mathcal{R}$. Let $L a b_{R}$ and $S$ be disjoint sets. By Definition 12 the flattened reaction system $\mathcal{A}$ of $\Delta$ is defined as follows: $\mathcal{A}=\left(S^{\prime}, A^{\prime}\right)$ where $S^{\prime}=\left\{[a, i] \mid a \in\left(S \cup L a b_{R}\right), 1 \leq i \leq n\right\}$. To each $r c$-reaction $\rho:\left(R_{\rho}, I_{\rho}, P_{\rho}\right) ; \operatorname{target}(\rho)$ in $\mathcal{R}$ and for each $i, 1 \leq i \leq n$, there is a reaction $\left(\rho^{\prime}, i\right):\left(\left\{\left[l_{\rho}, i\right]\right\} \cup\left\{[a, i] \mid a \in R_{\rho}\right\},\left\{[b, i] \mid b \in I_{\rho}\right\},\left\{[c, i] \mid \in P_{\rho}\right\} \cup\left\{\left[l_{\rho}, k\right] \mid k \in\right.\right.$ target $(\rho)\})$. $\mathcal{A}$ has no more reactions.

Let us define $\operatorname{cdcR}(\mathrm{p})$ system $\Delta^{\prime}$ as follows. Let $\Delta^{\prime}=\left(S^{\prime}, A_{1}^{\prime}, \ldots, A_{n}^{\prime}\right), n \geq 1$, where $S^{\prime}=\{[a, i] \mid a \in S, 1 \leq i \leq n\} \cup\left\{\left[l_{\rho}, i\right] \mid \rho \in \mathcal{R}, 1 \leq i \leq n\right\}$. Let $A_{i}^{\prime}$ be defined as follows: for $\rho:\left(R_{\rho}, I_{\rho}, P_{\rho}\right) ; \operatorname{target}(\rho)$ in $\mathcal{R}$ we define pc-reaction $\rho^{\prime}:\left(\left\{l_{\rho}\right\} \cup R_{\rho}, I_{\rho},\left\{[c, i] \mid c \in P_{\rho}\right\} \cup\left\{l_{\rho}(j) \mid j \in \operatorname{target}(\rho)\right\}\right)$.

It is easy to see that after performing the $p c$-reaction $\rho^{\prime}$, elements of $S$ that are products in $\rho$ stay with the component, while the label of $\rho, l_{\rho}$, is communicated to those components that are given as targets of $\rho$ in $\Delta$.

Now let us construct the flattened version of $\Delta^{\prime}$, given in Definition 7 , denoted by $\mathcal{A}^{\prime}$. Then for each reaction $\rho^{\prime}$ of $\Delta^{\prime}$, see above, we obtain reaction $\left(\rho^{\prime \prime}, i\right)$ : $\left(\left\{\left[l_{\rho}, i\right]\right\} \cup\left\{[a, i] \mid a \in R_{\rho}\right\},\left\{[b, i] \mid b \in I_{\rho}\right\},\left\{[c, i] \mid c \in P_{\rho}\right\} \cup\left\{\left[l_{\rho}, k\right] \mid k \in\right.\right.$ target $(\rho)\})$. Then it is easy to see that $\mathcal{A}^{\prime}=\mathcal{A}$ holds.

\section{Conclusions}

In this paper we introduced new variants of networks of reaction systems where the components communicate with each other by sending products or reactions. We proved that these networks can be represented by single reaction systems (flattened reaction systems), and discussed some aspects of communication in these networks. We pointed out a connection between the occurrence of a reactant (a reaction) at some component of the $\operatorname{cdcR}(\mathrm{p})$ system $(\operatorname{cdcR}(\mathrm{r})$ system) at some step of the operation and the occurrence of the corresponding reactant in the same step of the operation of the corresponding flattened reaction system. Occurrence problems and their complexity for reaction systems have been studied in $[12,10]$ and were shown to be NP-complete (or PSPACE-complete) problems, depending on how the problem is formulated. These studies and results can be interpreted in terms of $\operatorname{cdcR}(\mathrm{p})$ systems $(\operatorname{cdcR}(\mathrm{r}))$ systems. In the future, we plan to study the connections between $\mathrm{R}$ systems and $\mathrm{P}$ systems (see, for example $[1,2])$. Further types of direct communication protocols, dynamic behavior would also be of interest to investigate. 


\section{Acknowledgment}

The authors thank the reviewers for their valuable comments. The work of Erzsébet Csuhaj-Varjú was supported by the National Research, Development, and Innovation Office - NKFIH, Hungary, Grant no. K 120558. The work of Pramod Kumar Sethy was supported by project " Integrált kutatói utánpótlásképzési program az informatika és számítástudomány diszciplináris területein", EFOP 3.6.3-VEKOP-16-2017-00002, a project supported by the European Union and co-funded by the European Social Fund.

\section{References}

1. Alhazov, A.: P systems without multiplicities of symbol-objects. Inf. Process. Lett. 100(3), 124-129 (2006)

2. Alhazov, A., Aman, B., Freund, R., Ivanov, S.: Simulating R systems by P systems. In: Leporati, A., Rozenberg, G., Salomaa, A., Zandron, C. (eds.) Membrane Computing - 17th International Conference, CMC 2016, Milan, Italy, July 25-29, 2016, Revised Selected Papers. Lecture Notes in Computer Science, vol. 10105, pp. 51-66. Springer (2016)

3. Bottoni, P., Labella, A., Rozenberg, G.: Reaction systems with influence on environment. J. Membr. Comput. 1(1), 3-19 (2019)

4. Bottoni, P., Labella, A., Rozenberg, G.: Networks of reaction systems. Int. J. Found. Comput. Sci. 31(1), 53-71 (2020)

5. Castellanos, J., Martín-Vide, C., Mitrana, V., Sempere, J.M.: Solving NP-complete problems with networks of evolutionary processors. In: Mira, J., Prieto, A. (eds.) Connectionist Models of Neurons, Learning Processes and Artificial Intelligence, 6th International Work-Conference on Artificial and Natural Neural Networks, IWANN 2001 Granada, Spain, June 13-15, 2001, Proceedings, Part I. Lecture Notes in Computer Science, vol. 2084, pp. 621-628. Springer (2001)

6. Csuhaj-Varjú, E., Kelemen, J., Păun, G.: Grammar systems with wave-like communication. Comput. Artif. Intell. 15(5) (1996)

7. Csuhaj-Varjú, E., Salomaa, A.: Networks of parallel language processors. In: Păun, G., Salomaa, A. (eds.) New Trends in Formal Languages - Control, Cooperation, and Combinatorics (to Jürgen Dassow on the occasion of his 50th birthday). Lecture Notes in Computer Science, vol. 1218, pp. 299-318. Springer (1997)

8. Ehrenfeucht, A., Rozenberg, G.: Basic notions of reaction systems. In: Calude, C., Calude, E., Dinneen, M.J. (eds.) Developments in Language Theory, 8th International Conference, DLT 2004, Auckland, New Zealand, December 13-17, 2004, Proceedings. Lecture Notes in Computer Science, vol. 3340, pp. 27-29. Springer (2004)

9. Ehrenfeucht, A., Rozenberg, G.: Reaction systems. Fundam. Informaticae 75(1-4), 263-280 (2007)

10. Formenti, E., Manzoni, L., Porreca, A.E.: On the complexity of occurrence and convergence problems in reaction systems. Nat. Comput. 14(1), 185-191 (2015)

11. Hopcroft, J.E., Motwani, R., Ullman, J.D.: Introduction to automata theory, languages, and computation, 3rd Edition. Pearson international edition, AddisonWesley (2007)

12. Salomaa, A.: Functional constructions between reaction systems and propositional logic. Int. J. Found. Comput. Sci. 24(1), 147-160 (2013) 